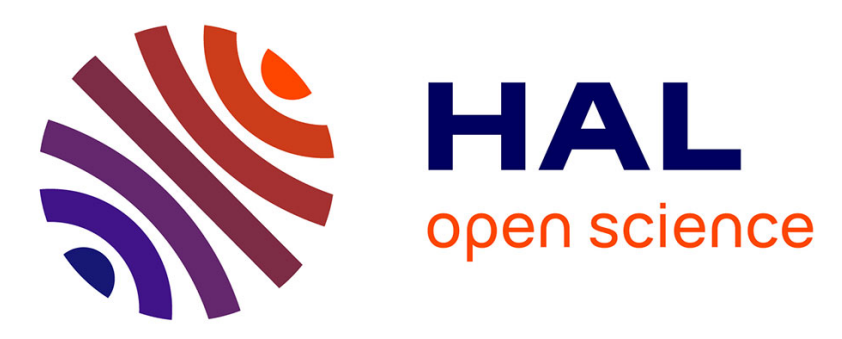

\title{
Multifractal-based texture segmentation using variational procedure
}

Jordan Frecon, Nelly Pustelnik, Herwig Wendt, Laurent Condat, Patrice Abry

\section{To cite this version:}

Jordan Frecon, Nelly Pustelnik, Herwig Wendt, Laurent Condat, Patrice Abry. Multifractal-based texture segmentation using variational procedure. IEEE 12th Image, Video, and Multidimensional Signal Processing Workshop (IVMSP 2016), Jul 2016, Bordeaux, France. pp.1 - 5, 10.1109/IVMSPW.2016.7528187 . hal-01398865

\section{HAL Id: hal-01398865 https://hal.science/hal-01398865}

Submitted on 18 Nov 2016

HAL is a multi-disciplinary open access archive for the deposit and dissemination of scientific research documents, whether they are published or not. The documents may come from teaching and research institutions in France or abroad, or from public or private research centers.
L'archive ouverte pluridisciplinaire HAL, est destinée au dépôt et à la diffusion de documents scientifiques de niveau recherche, publiés ou non, émanant des établissements d'enseignement et de recherche français ou étrangers, des laboratoires publics ou privés. 


\title{
MULTIFRACTAL-BASED TEXTURE SEGMENTATION USING VARIATIONAL PROCEDURE
}

\author{
Jordan Frecon ${ }^{1}$, Nelly Pustelnik ${ }^{1}$, Herwig Wendt ${ }^{2}$, Laurent Condat ${ }^{3}$, Patrice Abry ${ }^{1}$, \\ ${ }^{1}$ Univ Lyon, Ens de Lyon, Univ Lyon 1, CNRS, Laboratoire de Physique, F-69342 Lyon, France. firstname. lastname@ens-lyon. fr \\ 2 IRIT, CNRS UMR 5505, INP-ENSEEIHT, F-31062 Toulouse, France. herwig.wendteirit.fr \\ ${ }^{3}$ Univ Grenoble Alpes, CNRS, GIPSA-Lab, F-38000 Grenoble, France. laurent. condategipsa-lab.grenoble-inp.fr
}

\begin{abstract}
The present contribution aims at segmenting a scale-free texture into different regions, characterized by an a priori (unknown) multifractal spectrum. The multifractal properties are quantified using multiscale quantities $C_{1, j}$ and $C_{2, j}$ that quantify the evolution along the analysis scales $2^{j}$ of the empirical mean and variance of a nonlinear transform of wavelet coefficients. The segmentation is performed jointly across all the scales $j$ on the concatenation of both $C_{1, j}$ and $C_{2, j}$ by an efficient vectorial extension of a convex relaxation of the piecewise constant Potts segmentation problem. We provide comparisons with the scalar segmentation of the Hölder exponent as well as independent vectorial segmentations over $C_{1}$ and $C_{2}$.
\end{abstract}

Index Terms - Local regularity, multifractal spectrum, segmentation, convex optimization, wavelet leaders

\section{INTRODUCTION}

Recent contributions in image processing highlighted the need of segmentation techniques for scale-free textures analysis $[1,2,3]$. The possible applications go from texture medical images such as the bone study [4] to art investigations $[5,6]$.

Scale-free behavior is captured with local regularity, technically measured via the concept of Hölder exponent [7]. First studies based on the estimation of this quantity can be traced back to [8] where local regularity was assumed homogeneous throughout the image (i.e., monofractal). Recent contributions have considered a more realistic model, where the local regularity may be heterogeneous throughout the image (i.e, piecewise monofractal). This further increases the complexity of the estimation procedure since it additionnaly amounts in segmenting the image into a priori unknown regions where the local regularity can be considered homogeneous.. Among numerous techniques for image segmentation, some efficient variational approaches have recently been designed relying on the use of the total variation $[9,1,3,10]$. The good results of

Work supported by GdR 720 ISIS under the junior research project GALILEO, ANR AMATIS grant \#112432, 2010-2014, and CNRS Imag' in project under grant 2015OPTIMISME. these estimation and segmentation techniques (on simulated and real data) thus pave the way for considering more complex piecewise scale-free models. This is the subject of the present contribution.

We aim to go further by proposing a segmentation method for piecewise multifractal processes analysis, thus allowing a richer modeling of real-world textures. The multifractal formalism detailled Section 2 relies on the local estimation of multiscale quantities in place of the local regularity. It is combined with a joint vectorial segmentation procedure whose algorithmic solution is briefly summarized in Section 3. Estimation performance on synthetic results is reported in Section 4. Comparisons with state-of-the-art methods are also provided.

\section{MULTIFRACTAL ANALYSIS}

Piecewise mono/multi-fractal. We denote $X=\left(X_{\ell}\right)_{1 \leq \ell \leq N}$ the scale-free image to analyze, which has $N$ pixels. Its local regularity can be quantified by the Hölder exponent [7] denoted by $h=\left(h_{\underline{\ell}}\right)_{1 \leq \underline{\ell} \leq N}$. While large $h_{\underline{\ell}}$ points to a locally smooth portion of the field, low $h_{\underline{\ell}}$ indicates local high irregularity. Two examples of $X$ are provided in Figure 1(b)-(c).

On the one hand, in Figure 1(b), $X$ models a piecewise monofractal process having two different values of $h_{\underline{\ell}}$, i.e.

$$
\begin{array}{ll}
\left(\forall \underline{\ell} \in \Omega_{A}\right) & h_{\underline{\ell}}=h_{A}, \\
\left(\forall \underline{\ell} \in \Omega_{B}\right) & h_{\underline{\ell}}=h_{B},
\end{array}
$$

with $h_{A}<h_{B}$ and $\Omega=\Omega_{A} \cup \Omega_{B}$ being separable in two distinct areas, i.e., $\Omega_{A} \cap \Omega_{B}=\emptyset$, according to the mask presented in Figure 1 (a). A smoother behavior is thus observed for $\underline{\ell} \in \Omega_{A}$ than for $\underline{\ell} \in \Omega_{B}$.

On the other hand, in Figure 1 (c), $X$ models a piecewise multifractal process where $h_{\underline{\ell}}$ may locally vary both within $\Omega_{A}$ and $\Omega_{B}$. Therefore, the multifractal spectrum $\mathcal{D}(h)$, which describes local regularity fluctuations, is not reduced to a Dirac (cf. e.g., $[7,11,12]$ for details), and

$$
\begin{aligned}
& \left(\forall \underline{\ell} \in \Omega_{A}\right) \quad h_{\underline{\ell}} \text { can be described by } \mathcal{D}_{A}(h) \\
& \left(\forall \underline{\ell} \in \Omega_{B}\right) \quad h_{\underline{\ell}} \text { can be described by } \mathcal{D}_{B}(h) .
\end{aligned}
$$




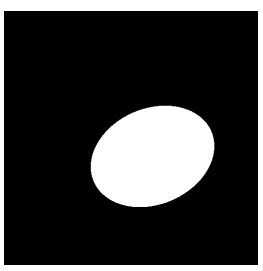

(a) Mask

$\Omega_{A}$ : white area

$\Omega_{B}$ : black area

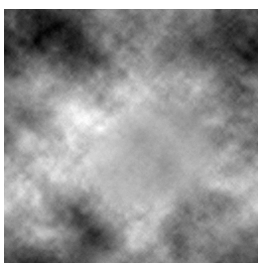

(b) $X$ piecewise monofractal

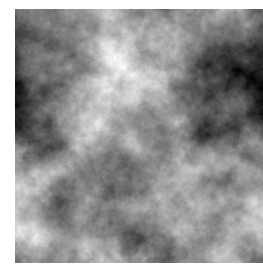

(c) $X$ piecewise multifractal
Fig. 1. Examples of scale-free textures.

The aim of multifractal analysis is to estimate $\mathcal{D}(h)$. For practical purposes, the multifractal spectrum can often be approximated as a parabola: $\mathcal{D}(h)=2+\left(h-c_{1}\right)^{2} /\left(2 c_{2}\right)$. Here, we follow the efficient procedure proposed in [12] based on wavelet-leader coefficients [7].

Wavelet-leader coefficients. We denote $d_{j, \underline{k}}^{(m)}=\left\langle X, \psi_{j, \underline{k}}^{(m)}\right\rangle$ the ( $L^{1}$-normalized) 2D discrete wavelet coefficients of $X$ at location $\underline{k}=2^{-j} \underline{\ell}$, at scale $2^{j}$ with $j \in\{1, \ldots, J\}$, and where $m$ stands for the horizontal/vertical/diagonal subband. For a detailed definition of the 2D-DWT, readers are referred to e.g., [13]. The wavelet leader coefficient $L_{j, \underline{k}}$, located around position $\underline{\ell}=2^{j} \underline{k}$, is defined as the local supremum of all wavelet coefficients taken within a spatial neighborhood across all finer scales $2^{j^{\prime}} \leq 2^{j}$, that is,

$$
L_{j, \underline{k}}=\sup _{\substack{m=1,2,3, \lambda_{j^{\prime}, \underline{k}} \subset \Lambda_{j, \underline{k}}}}\left|d_{j^{\prime}, \underline{k}^{\prime}}^{(m)}\right|,
$$

with $\lambda_{j, \underline{k}}=\left[\underline{k} 2^{j},(\underline{k}+1) 2^{j}\right)$, and $\Lambda_{j, \underline{k}}=\bigcup_{p \in\{-1,0,1\}^{2}} \lambda_{j, \underline{k}+p}$. An illustration is provided in Figure 2 where $L_{j, \underline{k}}$ is indicated with a black cross and the neighborhood $\Lambda_{j, \underline{k}}$ is displayed in red.

Hölder exponent. The Hölder exponent can be obtained by a linear regression of wavelet-leader coefficients as follows:

$$
h_{\underline{k}}=\sum_{j \in\{1, \ldots, J\}} w_{j, \underline{k}} \log _{2} L_{j, \underline{k}},
$$

where the $w_{j, \underline{k}}$ model regression weights [11].

Multifractal spectrum. The multifractal spectrum can be obtained by multiscale quantities $C_{1, j} \in \mathbb{R}^{N}$ and $C_{2, j} \in \mathbb{R}^{N}$ defined as the sample estimates of the first and second cumulant of $\log _{2} L_{j}$ at each given scale $2^{j}$. It has been shown that $C_{1, j}$ and $C_{2, j}$ are related to the multifractal spectrum $\mathcal{D}(h)$ via the coefficients $c_{1}$ and $c_{2}$ as follows [12]:

$$
\begin{aligned}
& \mathbb{E} C_{1, j}=c_{1}^{0}+c_{1} \ln 2^{j}, \\
& \mathbb{E} C_{2, j}=c_{2}^{0}+c_{2} \ln 2^{j} .
\end{aligned}
$$

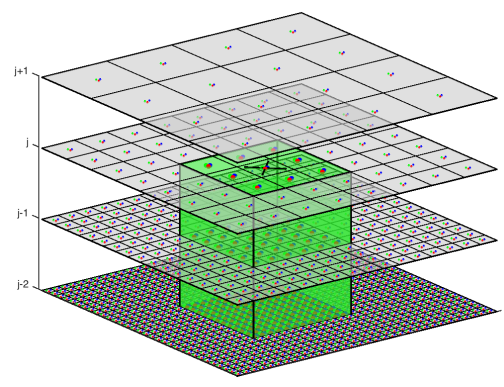

Fig. 2. waveleter-leader coefficients. The wavelet leader coefficient (black cross) is defined as the local supremum of all wavelet coefficients taken within a spatial neighborhood across all finer scales (red)

To evaluate changes in multifractal spectrum, one could naturally consider estimating $C_{1, j}$ and $C_{2, j}$ locally in a neighborhood of each pixel $\underline{\ell}$, estimate the corresponding local parameters $c_{1}$ and $c_{2}$, and then perform a vectorial segmentation of $\left(c_{1}, c_{2}\right)$. However, this relies on the strong assumption that real-world textures follow precisely the scaling behaviors prescribed in Eqs. (7) and (8) above. In the present contribution, it has been chosen to relax this requirement and to directly perform a vectorial segmentation over multiscale quantities $C_{1, j}$ and $C_{2, j}$, possibly smoothed.

\section{VECTORIAL SEGMENTATION}

We propose to follow segmentation procedure ideas that perform well for piecewise mono/multi-fractal estimation [2]. The vectorial segmentation procedure is based on a convex relaxation of the piecewise constant Potts segmentation problem $[9,14]$. Here we consider the extension to joint vectorial segmentation proposed in [9] for image labelling. In what follows, $Y=\left(Y_{m}\right)_{1 \leq m \leq M} \in \mathbb{R}^{N \times M}$ can either model $h$ (i.e., $M=1), C_{1}$ or $C_{2}$ (i.e., $M=J$ ), or their concatenation $\left(C_{1}, C_{2}\right)$ (i.e., $\left.M=2 J\right)$.

Problem formulation. The labeling procedure of $Y$ into $Q$ level sets can be formalized as a minimization problem where $Q+1$ binary images $\Theta=\left(\theta_{q}\right)_{1 \leq q \leq(Q+1)} \in \mathbb{R}^{N \times Q+1}$ are estimated such that

$$
\begin{aligned}
\min _{\Theta} \sum_{q=1}^{Q}\left(\theta_{q}-\theta_{q+1}\right)^{\top} & \sum_{m=1}^{M}\left(Y_{m}-v_{q, m}\right)^{2}+\lambda \sum_{q=1}^{Q} \operatorname{TV}\left(\theta_{q}-\theta_{q+1}\right) \\
\text { subject to } \quad & \left\{\begin{array}{l}
\theta_{1}=1 \\
\theta_{Q+1}=0 \\
1 \geq \theta_{2} \geq \ldots \geq \theta_{Q} \geq 0
\end{array}\right.
\end{aligned}
$$

with $\lambda>0$ and where TV denotes the usual total-variation penalization as defined in [15], i.e., for every $\theta \in \mathbb{R}^{N}$, 


$$
\operatorname{TV}(\theta)=\sum_{\underline{\ell}=1}^{N}\left\|(D \theta)_{\underline{\ell}}\right\|_{2},
$$

where $D \in \mathbb{R}^{2 N \times N}$ stands for the discrete horizontal/vertical difference operator and thus $(D \theta)_{\ell} \in \mathbb{R}^{2}$. The choice of $v_{q, m} \in \mathbb{R}$ will be discussed further.

The $Q$ resulting labelling areas $\left(\Omega_{1}, \ldots, \Omega_{Q}\right)$ are obtained from the binary images $\left(\theta_{q}-\theta_{q+1}\right)_{1 \leq q \leq Q}$ as follows:

$$
(\forall \underline{\ell} \in \Omega) \quad \theta_{q, \underline{\ell}}-\theta_{q+1, \underline{\ell}}=\left\{\begin{array}{l}
1, \text { if } \underline{\ell} \in \Omega_{q}, \\
0, \text { otherwise. }
\end{array}\right.
$$

The first term in (9) is a data fidelity term allowing to impose similar properties within each area $\Omega_{q}$. The second term imposes the regularity for each labelling area $\Omega_{q}$. The smaller is $\lambda$ the higher is the granularity of $\Omega_{q}$.

Algorithmic solution. In order to efficiently estimate $\Theta$ we use the algorithmic strategy proposed in [16] that consists in using a proximal splitting method coupled with an efficient strategy to compute the involved proximal operators. The reader could refer to $[9,16]$ for details regarding the algorithmic strategy for solving (9).

\section{EXPERIMENTS}

Experimental setting. Performance of the proposed segmentation procedure are assessed on synthetic data, numerically produced by inclusion of a $2 \mathrm{D}$ MRW patch $\Omega_{A}$ [17] into a 2D-MRW background $\Omega_{B}$ with different multifractal parameters. Patch and background (Figure 3 (b)) have been normalized to ensure that the local variance does not depend on the image location. An illustration is provided in Figure 3 (a) where the background parameters $\left(c_{1}, c_{2}\right)=(0.7,0.1)$ and the patch parameters $\left(c_{1}, c_{2}\right)=(0.4,0.005)$.

The wavelet leader coefficients are estimated using a standard 2D-DWT with orthonormal tensor product Daubechies mother wavelets with 2 vanishing moments.

In our simulation, we have set $Q=2, \lambda=1$ and $J=3$. For every component $m \in\{1, \ldots M\},\left(v_{q, m}\right)_{1 \leq q \leq Q}$ are initially chosen to be equally distributed between the minimum and maximum values of $Y_{m}$. Then, an alternate minimization of (9) and re-estimation of the $\left(v_{q, m}\right)_{1 \leq q \leq Q}$ is performed 5 times.

Scalar vs. vectorial segmentation. The scalar segmentation of $Y=h$, originally envisaged for the analysis of piecewise monofractal processes [3], is illustrated in Figure 3-(c)). We observe that it yields poor results. Indeed, since the process is multifractal, every $h$ of the support of $\mathcal{D}(h)$ is present in any open interval of the trajectory for finite resolution data. The local estimation of $h$ is hence not very meaningful. In addition, since $\mathcal{D}_{A}$ and $\mathcal{D}_{B}$ overlap, the sole feature $h$ can not permit to discriminate between $\Omega_{A}$ and $\Omega_{B}$. This limitation thus shows the need of investigating multiscale quantities related to the multifractal spectrum, namely $C_{1}$ and $C_{2}$. Such a strategy has been previously investigated in [2] with a different algorithmic solution involving a different segmentation for each component $m \in\{1, \ldots, M\}$.

Segmentation performance. For the same patch displayed in Figure 3 (b), we have considered eight configurations reported in Figure 4 and defined as follow. The top (resp. bottom) plots correspond to a large (resp. small) difference of $c_{1}$ between $\Omega_{A}$ and $\Omega_{B}$. The left (resp. right) plots model large (resp. small) difference of $c_{2}$ between $\Omega_{A}$ and $\Omega_{B}$. In addition, we have further investigated the impact of $c_{2}$ : results in orange correspond to two tight multifractal spectrum $\mathcal{D}_{A}$ and $\mathcal{D}_{B}$ (i.e., small $c_{2}$ on both $\Omega_{A}$ and $\Omega_{B}$ ) whereas the blue one models one tight $\mathcal{D}_{A}$ (i.e., small $c_{2}$ on $\Omega_{A}$ ) and a widespread $\mathcal{D}_{B}$ (i.e., large $c_{2}$ on $\Omega_{B}$ ). Estimation performance are quantified in terms of misclassified pixels percentage over 20 realizations for all the different inputs $Y$.

Unsurprisingly, using $Y=h$ always leads to poor segmentation performance. In addition, all these experiments reproduce the expected behavior that the larger is the difference of $c_{1}$ (resp. $c_{2}$ ) between $\Omega_{A}$ and $\Omega_{B}$, the better are the segmentation performance associated to $Y=C_{1}$ (resp. $Y=C_{2}$ ). However, a closer inspection shows that a larger difference in $c_{1}$ does not necessarily lead to better segmentation results for $Y=C_{2}$ (see the orange line in both left plots). Overall, we observe that there is always an interest in combining the information of both $C_{1}$ and $C_{2}$.

Finally, it is worth noticing that the scalar segmentation of $h$ is only 2 times faster than the vectorial ones either based on $C_{1}, C_{2}$ or $\left(C_{1}, C_{2}\right)$. Experimentally, it takes less than 1 minute per image of size $N=2^{9} \times 2^{9}$.

\section{CONCLUSIONS AND PERSPECTIVES}

In this work, we have designed an analysis procedure for dealing with piecewise multifractal processes. We have shown the need of considering multiscale quantities $C_{1}$ and $C_{2}$ rather than the sole Hölder exponent usually considered for piecewise monofractal processes. An efficient joint vectorial segmentation procedure is proposed and yields satisfactory performance when applied to $\left(C_{1}, C_{2}\right)$.

However, the proposed segmentation procedure rely on the strong assumption that $\theta_{q, 1}-\theta_{q+1,1}=\ldots=\theta_{q, M}-$ $\theta_{q+1, M}$. Therefore, the performance are very sensitive to the arbitrary choice and order of the level sets $v_{q, m}$. In order to alleviate this limitation, different regularization terms are under current investigation to provide a more flexible joint vectorial segmentation strategy. 


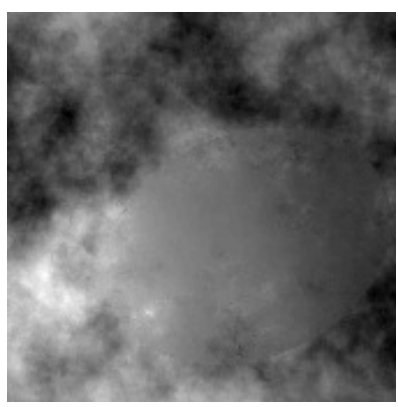

(a) Piecewise multifractal $X$

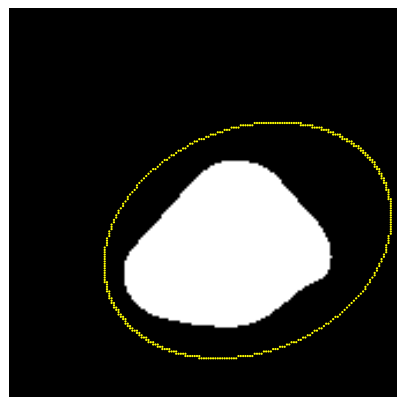

(d) Segmentation based on $C_{1}$ Misclassified pixels: $9.12 \%$

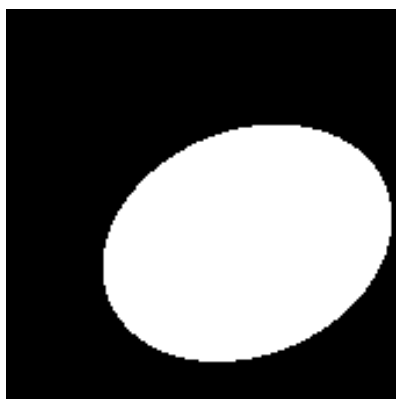

(b) Mask

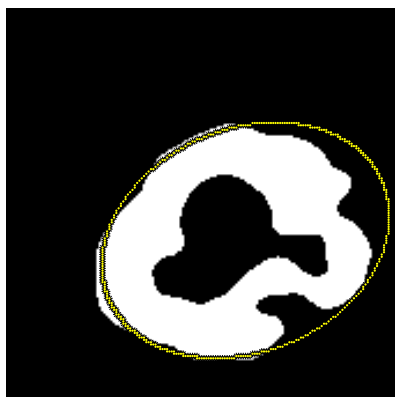

(e) Segmentation based on $C_{2}$ Misclassified pixels: $5.14 \%$

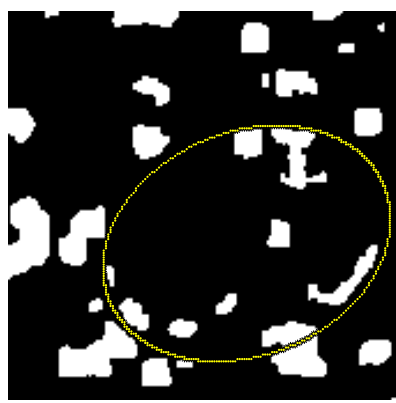

(c) Segmentation based on $h$ Misclassified pixels: $18.0 \%$

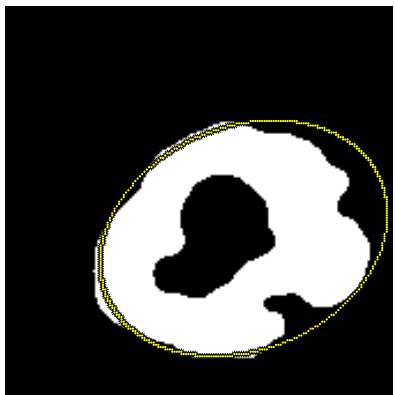

(f) Segmentation based on $\left(C_{1}, C_{2}\right)$ Misclassified pixels: $3.84 \%$

Fig. 3. Illustration of the segmentation results. The piecewise multifractal image $X$ is presented in (a) and has been generated from the mask (b). The scalar segmentation result obtained from $h$ is displayed in (c). The vectorial segmentation results based on $C_{1}, C_{2}$ and $\left(C_{1}, C_{2}\right)$ are respectively presented in (d), (e) and (f).

\section{REFERENCES}

[1] C. Nafornita, A. Isar, and J. D. B. Nelson, "Semilocal Hurst estimation via generalised lasso and dualtree complex wavelets," in Proc. Int. Conf. Image Process., Paris, France, Oct. 27-30 2014, pp. 2689-2693.

[2] J. Frecon, N. Pustelnik, H. Wendt, and P. Abry, "Multivariate optimization for multifractal-based texture segmentation," in Proc. Int. Conf. Image Process., Quebec City, Canada, Sept, 27-30 2015.

[3] N. Pustelnik, H. Wendt, P. Abry, and N Dobigeon, "Local regularity, wavelet leaders and total variation based procedures for texture segmentation,", Tech. Rep., arXiv:1504.05776, 2016.

[4] C.L. Benhamou, S. Poupon, E. Lespessailles, S. Loiseau, R. Jennane, V. Siroux, W. J. Ohley, and L. Pothuaud, "Fractal analysis of radiographic trabecular bone texture and bone mineral density: two complementary parameters related to osteoporotic fractures," J. Bone Miner. Res., vol. 16, no. 4, pp. 697-704, 2001.

[5] P. Abry, S. Jaffard, and H. Wendt, "When Van Gogh meets Mandelbrot: Multifractal classification of paint- ing's texture," Signal Process., vol. 93, no. 3, pp. 554$572,2013$.

[6] C. R. Johnson, P. Messier, W. A. Sethares, A. G. Klein, C. Brown, P. Klausmeyer, P. Abry, S. Jaffard, H. Wendt, S. G. Roux, N. Pustelnik, N. van Noord, L. van der Maaten, E. Postma, J. Coddington, L. A. Daffner, H. Murata, H. Wilhelm, S. Wod, and M. Messier, "Pursuing automated classification of historic photographic papers from raking light photomicrographs.," Journal of the American Institute for Conservation, vol. 53, no. 3, pp. 159-170, 2014.

[7] S. Jaffard, "Wavelet techniques in multifractal analysis," in Fractal Geometry and Applications: A Jubilee of Benoît Mandelbrot, M. Lapidus and M. van Frankenhuijsen Eds., Proceedings of Symposia in Pure Mathematics, M. Lapidus and M. van Frankenhuijsen, Eds. 2004, vol. 72, pp. 91-152, AMS.

[8] S. G. Roux, A. Arneodo, and N. Decoster, "A waveletbased method for multifractal image analysis. III. Applications to high-resolution satellite images of cloud structure," Eur. Phys. J. B, vol. 15, no. 4, pp. 765-786, 2000 .

[9] A. Chambolle, D. Cremers, and T. Pock, "A convex 

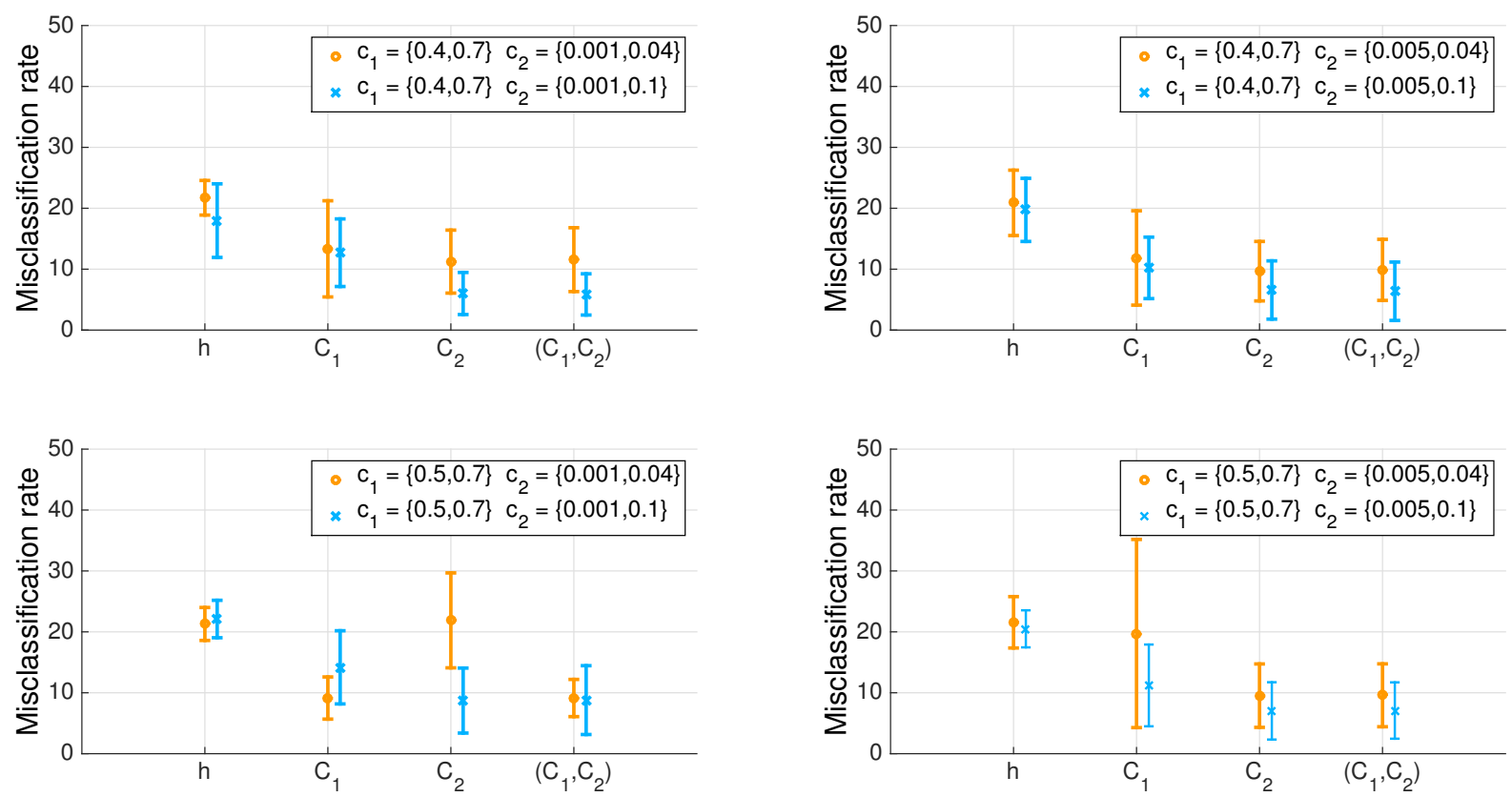

Fig. 4. Segmentation performance. Comparison of the segmentation performance is quantified in terms of mean misclassification rate depending on the choice of the input $Y$ represented along the $x$-axis (error bar indicate the standard deviation). Each plot represent two configurations (blue and orange) of $c_{1}$ and $c_{2}$ on $\Omega_{A}$ and $\Omega_{B}$.

approach to minimal partitions," SIAM J. Imaging Sci., vol. 5, no. 4, pp. 1113-1158, 2012.

[10] J.D.B. Nelson, C. Nafornta, and A. Isar, "Semilocal scaling exponent estimation with box-penalty constraints and total-variation regularization," IEEE Trans. Image Process., vol. 25, no. 6, pp. 3167-3181, Apr. 2016.

[11] H. Wendt, P. Abry, and S. Jaffard, "Bootstrap for empirical multifractal analysis," IEEE Signal Process. Mag., vol. 24, no. 4, pp. 38-48, Jul. 2007.

[12] H. Wendt, S.G. Roux, P. Abry, and S. Jaffard, "Wavelet leaders and bootstrap for multifractal analysis of images," Signal Proces., vol. 89, pp. 1100-1114, 2009.

[13] S. Mallat, A wavelet tour of signal processing, Academic Press, San Diego, USA, 1997.

[14] M. Storath and A. Weinmann, "Fast partitioning of vector-valued images," SIAM J. Imaging Sci., vol. 7, no. 3, pp. 1826-1852, 2014.

[15] A. Chambolle, "An algorithm for total variation minimization and applications," J. Math. Imag. Vis., vol. 20, no. 1-2, pp. 89-97, Jan. 2004.
[16] L. Condat and N. Pustelnik, "Segmentation d'image par optimisation proximale," in Proc. GRETSI, Lyon, France, September 8-11, 2015, in French.

[17] Raoul Robert, Vincent Vargas, et al., "Gaussian multiplicative chaos revisited," The Annals of Probability, vol. 38, no. 2, pp. 605-631, 2010. 